\title{
Asymptotic Formulae for Weight Numbers of the Sturm - Liouville Boundary Problem on a Star-shaped Graph
}

\section{A. Kuznetsova}

Maria A. Kuznetsova, Saratov State University, https://orcid.org/0000-0003-1083-0799, 83, Astrakhanskaya Str., 410012, Saratov, Russia, mk680970@gmail.com

In this article the Sturm - Liouville boundary value problem on the graph $\Gamma$ of a special structure is considered. The graph $\Gamma$ has $m$ edges, joined at one common vertex, and $m$ vertices of degree 1 . The boundary value problem is set by the Sturm - Liouville differential expression with real-valued potentials, the Dirichlet boundary conditions, and the standard matching conditions. This problem has a countable set of eigenvalues. We consider the so-called weight numbers, being the residues of the diagonal elements of the Weyl matrix in the eigenvalues. These elements are meromorphic functions with simple poles which can be only the eigenvalues. We note that the considered weight numbers generalize the weight numbers of Sturm - Liouville operators on a finite interval, equal to the reciprocals to the squared norms of eigenfunctions. These numbers together with the eigenvalues play a role of spectral data for unique reconstruction of operators. We obtain asymptotic formulae for the weight numbers using the contour integration, and in the case of the asymptotically close eigenvalues the formulae are got for the sums. The formulae can be used for the analysis of inverse spectral problems on the graphs.

Key words: Sturm - Liouville boundary problem, asymptotic formulae, weight numbers, star-shaped graph.

DOI: $10.18500 / 1816-9791-2018-18-1-40-48$

\section{INTRODUCTION}

Consider the graph $\Gamma$ which consists of $m$ edges $e_{j}, m \geqslant 2, j=\overline{1, m}$, joined at a common vertex. Let the graph $\Gamma$ be parametrized so that $x_{j} \in[0, \pi]$ where the parameter $x_{j}$ corresponds to the edge $e_{j}$, the parameter $x_{j}=0$ in the boundary vertex and $x_{j}=\pi$ in the common vertex, $j=\overline{1, m}$. We call $\Gamma$ a star-shaped graph.

A function on the graph is a vector function

$$
y=\left[y_{j}\left(x_{j}\right)\right]_{j=1}^{m},
$$

where the components $y_{j}$ are functions on the edges $e_{j}$ correspondingly, $y_{j}\left(x_{j}\right) \in W_{2}^{2}[0, \pi], j=\overline{1, m}$.

We denote by $g^{\prime}$ differentiation of the function $g$ with respect to the first argument. Consider the differential expression

$$
l y:=\left[-y_{j}^{\prime \prime}\left(x_{j}\right)+q_{j}\left(x_{j}\right) y_{j}\left(x_{j}\right)\right]_{j=1}^{m} .
$$

Then the Sturm-Liouville boundary value problem on the graph can be written as follows:

$$
\begin{gathered}
l y=\lambda y, \\
y_{j}(0)=0, \quad j=\overline{1, m}, \\
\sum_{j=1}^{m} y_{j}^{\prime}(\pi)=0, \\
y_{1}(\pi)=y_{2}(\pi)=\ldots=y_{m}(\pi),
\end{gathered}
$$


where $\lambda$ is the spectral parameter, the equalities (3) are Dirichlet conditions, and (4)-(5) are the standard matching conditions. In (1) the functions $q_{j}\left(x_{j}\right)$ are called potentials, $q_{j}\left(x_{j}\right) \in L_{2}[0, \pi], q_{j}\left(x_{j}\right) \in \mathbb{R}$.

The differential operator $L$, given by the differential expression (1) and the conditions (3)-(5), is self-adjoint in the corresponding Hilbert space (see [1] for details).

Since the differential operators on the graphs have applications in physics, chemistry, nano-technology, they are studied actively (see $[2,3]$ ). In the article we obtain asymptotic formulae for weight numbers of the problem (2)-(5) which generalize the weight numbers on a finite interval [4, Chapter 1]. Those asymptotic formulae can be applied for studying of inverse spectral problems for differential operators on graphs. Weight numbers together with eigenvalues have been used for reconstruction of the potentials of the Sturm - Liouville operators on graphs, e.g., in $[5,6]$.

The difficult case is when the eigenvalues are asymptotically close though not multiple. The asymptotic formulae are got by using the integration over the contours, containing the asymptotically close eigenvalues, in the plain of the spectral parameter.Thus, the asymptotic formulae are obtained for the sums of the weight numbers, as it has been done in [7] for the weight matrices for the matrix Sturm - Liouville operator.

\section{PRELIMINARIES}

In this section we introduce a characteristic function of the operator $L$, the zeros of which coincide with the eigenvalues. We also provide auxiliary results from $[8,9]$, related to the eigenvalues of $L$.

The conditions (4)-(5) can be written as follows:

$$
V(y):=H y^{\prime}(\pi)+h y(\pi)=0,
$$

where $H$ and $h$ are $m \times m$ matrices :

$$
H=\left(\begin{array}{cccccc}
1 & 1 & 1 & \ldots & 1 & 1 \\
0 & 0 & 0 & \ldots & 0 & 0 \\
\vdots & \vdots & \vdots & \ddots & \vdots & \vdots \\
0 & 0 & 0 & \ldots & 0 & 0 \\
0 & 0 & 0 & \ldots & 0 & 0
\end{array}\right), \quad h=\left(\begin{array}{cccccc}
0 & 0 & 0 & \ldots & 0 & 0 \\
1 & -1 & 0 & \ldots & 0 & 0 \\
0 & 1 & -1 & \ldots & 0 & 0 \\
\vdots & \vdots & \vdots & \ddots & \vdots & \vdots \\
0 & 0 & 0 & \ldots & 1 & -1
\end{array}\right)
$$

For each fixed $j=\overline{1, m}$ let $S_{j}(x, \lambda)$ and $C_{j}(x, \lambda)$ be the solutions of the Cauchy problems

$$
\begin{array}{ll}
-S_{j}^{\prime \prime}(x, \lambda)+q_{j}(x) S_{j}(x, \lambda)=\lambda S_{j}(x, \lambda), & S_{j}(0, \lambda)=S_{j}^{\prime}(0, \lambda)-1=0 \\
-C_{j}^{\prime \prime}(x, \lambda)+q_{j}(x) C_{j}(x, \lambda)=\lambda C_{j}(x, \lambda), & C_{j}(0, \lambda)-1=C_{j}^{\prime}(0, \lambda)=0 .
\end{array}
$$

Let the number $\rho$ be such that

$$
\lambda=\rho^{2}, \quad \arg \rho \in\left(-\frac{\pi}{2}, \frac{\pi}{2}\right] .
$$


The functions $S_{j}(x, \lambda), C_{j}(x, \lambda)$ satisfy the Volterra integral equations

$$
\begin{aligned}
& S_{j}(x, \lambda)=\frac{\sin \rho x}{\rho}+\int_{0}^{x} \frac{\sin \rho(x-t)}{\rho} q_{j}(t) S_{j}(t, \lambda) d t, \\
& C_{j}(x, \lambda)=\cos \rho x+\int_{0}^{x} \frac{\sin \rho(x-t)}{\rho} q_{j}(t) C_{j}(t, \lambda) d t .
\end{aligned}
$$

Put $\tau:=\operatorname{Im} \rho$. One can obtain the following asymptotic formulae from (7),(8) as $\rho \rightarrow \infty$ :

$$
\begin{gathered}
S_{j}(x, \rho)=\frac{\sin \rho x}{\rho}+\int_{0}^{x} \frac{\sin \rho(x-t)}{\rho^{2}} \sin \rho t q_{j}(t) d t+ \\
+\int_{0}^{x} \int_{0}^{t} \frac{\sin \rho(x-t) q_{j}(t)}{\rho^{3}} \sin \rho(t-\xi) q_{j}(\xi) \sin \rho \xi d \xi d t+O\left(\frac{e^{|\tau| x}}{\rho^{4}}\right), \\
+\int_{0}^{x} \int_{0}^{t} \frac{\cos \rho(x-t) q_{j}(t)}{\rho^{2}} \sin \rho(t-\xi) q_{j}(\xi) \sin \rho \xi d \xi d t+O\left(\frac{e^{|\tau| x}}{\rho^{3}}\right), \\
+\int_{0}^{x} \int_{0}^{t} \frac{\sin \rho(x-t) q_{j}(t)}{\rho^{2}} \sin \rho(t-\xi) q_{j}(\xi) \cos \rho \xi d \xi d t+O\left(\frac{e^{|\tau| x}}{\rho^{3}}\right), \\
C_{j}^{\prime}(x, \rho)=-\rho \sin \rho x+\int_{0}^{x} \cos \rho(x-t) \cos \rho t q_{j}(t) d t+ \\
+\int_{0}^{x} \int_{0}^{t} \frac{\cos \rho(x-t) q_{j}(t)}{\rho} \sin \rho(t-\xi) q_{j}(\xi) \cos \rho \xi d \xi d t+O\left(\frac{e^{|\tau| x}}{\rho^{2}}\right) .
\end{gathered}
$$

We introduce matrix solutions of equation (2): $S(\lambda)=\operatorname{diag}\left\{S_{j}\left(x_{j}, \lambda\right)\right\}_{j=1}^{m}$ and $C(\lambda)=\operatorname{diag}\left\{C_{j}\left(x_{j}, \lambda\right)\right\}_{j=1}^{m}$.

Every eigenvalue of problem (2)-(5) corresponds to the zero of the following characteristic function $\Delta(\lambda)$ :

$$
\Delta(\lambda):=\operatorname{det} V(S(\lambda))
$$

As $S_{j}(\pi, \lambda), S_{j}^{\prime}(\pi, \lambda)$ are entire functions of $\lambda$, the function $\Delta(\lambda)$ is also entire. Reconstructing the determinant in (13), we obtain

$$
\Delta(\lambda)=\sum_{k=1}^{m}\left(S_{k}^{\prime}(\pi, \lambda) \prod_{\substack{j=1, j \neq k}}^{m} S_{j}(\pi, \lambda)\right)
$$

Lemma 1. The number $\lambda_{0}$ is an eigenvalue of problem (2)-(5) of multiplicity $k$ if and only if $\lambda_{0}$ is a zero of characteristic function of multiplicity $k$.

The statement of the Lemma 1 results from the self-adjointness of $L$ and is proved with the same technique as in [7, Lemma 3]. From the self-adjointness of $L$ it also follows that the eigenvalues of the boundary problem (2)-(5) are real.

Denote $\omega_{j}=\frac{1}{2} \int_{0}^{\pi} q_{j}(t) d t, f(z)=\prod_{j=1}^{m}\left(z-\omega_{j}\right)$. Let $z^{(j)}, j=\overline{1, m-1}$ be the zeros of $f^{\prime}(z), z^{(m)}=\sum_{j=1}^{m} \omega_{j} / m$. We will mean by $\left\{\kappa_{n}\right\}_{n=1}^{\infty}$ different sequences from $l^{2}$. Considering these designations, the following theorem can be formulated: 
Theorem 1. The operator L has a countable set of the eigenvalues. The eigenvalues can be enumerated in such way that the further formulae are satisfied:

$$
\begin{gathered}
\sqrt{\lambda_{n}^{(j)}}=n+\frac{z^{(j)}+\kappa_{n}}{n \pi}, \quad j=\overline{1, m-1}, \\
\sqrt{\lambda_{n}^{(m)}}=n-\frac{1}{2}+\frac{z^{(m)}+\kappa_{n}}{n \pi},
\end{gathered}
$$

where the square root is taken according the same rule as in (6).

The formulae (15), (16) with the remainders $o(1)$ are obtained in [1]; Theorem 1 is proved for real-valued potentials by V. Pivovarchick [8] (see also [9]).

Remark 1. The statement of the Theorem 1 is also correct under the conditions $q_{j}(x) \in \mathbb{C}, j=\overline{1, m}$, all $\left\{z^{(k)}\right\}_{k=1}^{m-1}$ are distinct.

\section{MAIN RESULTS}

In that chapter we define and study weight numbers based on the Weyl matrix.

Let $\Phi(\lambda)=\left\{\phi_{j k}\left(x_{j}, \lambda\right)\right\}_{j, k=1}^{m}$ be the matrix solution of (2) under the conditions $\left\{\phi_{j k}(0, \lambda)\right\}_{j, k=1}^{m}=I, V(\Phi)=0$. The matrix $M(\lambda)=\left\{-\phi_{j k}^{\prime}(0, \lambda)\right\}_{j, k=1}^{m}$ is called the Weyl matrix and generalize the notion of the Weyl function for differential operators on intervals (see [4]). Weyl functions and their generalizations are natural spectral characteristics, often used for reconstruction of operators. A system of $2 m$ columns of the matrix solutions $C(\lambda), S(\lambda)$ is fundamental, and one can show, that

$$
M(\lambda)=(V(S(\lambda)))^{-1} V(C(\lambda)) .
$$

In view of (17) the elements of the matrix $M(\lambda)=\left\{M_{k l}(\lambda)\right\}_{k, l=1}^{m}$ can be calculated as

$$
M_{k l}(\lambda)=\frac{1}{\Delta(\lambda)}\left(\prod_{\substack{j=1, j \neq k}}^{m} S_{j}(x, \lambda) C_{l}(x, \lambda)\right)^{\prime} \mid .
$$

The elements of the matrix $M(\lambda)$ are meromorphic functions, and their poles may be only zeros of the characteristic function $\Delta(\lambda)$. Moreover, analogously to [7, Lemma 3], we prove the following lemma.

Lemma 2. If the number $\lambda_{0}$ is a pole of $M_{k l}(\lambda)$, this pole is simple.

Proof. Let $\lambda_{0}$ be a zero of $\Delta(\lambda)$ of multiplicity $b$. By virtue of Theorem 1 there are exactly $b$ linearly independent eigenfunctions $\left\{y_{j}\right\}_{j=1}^{b}$ corresponding to $\lambda_{0}$. Denote by $K$ such invertible matrix that first $b$ columns of $S\left(\lambda_{0}\right) K$ are equal to $\left\{y_{j}\right\}_{j=1}^{b}$.

If $Y(\lambda)=S(\lambda) K$, then $S(\lambda)=Y(\lambda) K^{-1}$, and

$$
M(\lambda)=K[V(Y(\lambda))]^{-1} V(C(\lambda)) .
$$


It is sufficient to prove that for any element of $A(\lambda)$ the number $\lambda_{0}$ can not be a pole of order greater than 1 , where $A(\lambda)=[V(Y(\lambda))]^{-1} V(C(\lambda))$. If $A(\lambda)=\left\{A_{s l}(\lambda)\right\}_{s, l=1}^{m}$, then

$$
A_{s l}(\lambda)=\frac{\operatorname{det}\left[V\left(Y_{1}(\lambda)\right), \ldots, V\left(Y_{s-1}(\lambda)\right), V\left(C_{l}(\lambda)\right), V\left(Y_{s+1}(\lambda)\right), \ldots, V\left(Y_{m}(\lambda)\right)\right]}{\operatorname{det} V(Y(\lambda))} .
$$

The number $\lambda_{0}$ is zero of the numerator of multiplicity not less then $b-1$. From that the statement of the theorem follows.

We introduce the constants $\alpha_{j n}^{k}=\underset{\lambda=\lambda_{n}^{(j)}}{\operatorname{Res}} M_{k k}(\lambda)$ which are called weight numbers. We also mean by $\left\{\kappa_{n}(z)\right\}_{n=1}^{\infty}$ different sequences of continuous functions such that

$$
\sum_{n=1}^{\infty} \max _{|z| \leqslant R}\left|\kappa_{n}(z)\right|^{2}<\infty
$$

where

$$
R=2+\max _{s=1, m}\left|z^{(s)}\right| .
$$

The main results of the paper are stated in the following two theorems.

Theorem 2. Let the eigenvalues of $L$ be enumerated as in Theorem $1, k=\overline{1, m}$. Then

$$
\begin{gathered}
\sum_{j \in I(n)} \alpha_{j n}^{k}=\frac{2 n^{2}}{m \pi}\left(m-1+\frac{\kappa_{n}}{n}\right), \\
\alpha_{m s}^{k}=\frac{\left(n-\frac{1}{2}\right)^{2}}{m \pi}\left(2+\frac{\kappa_{n}}{n}\right),
\end{gathered}
$$

where

$$
I(n)=\bigcup_{j=1}^{m-1}\left\{\min \left\{s: \lambda_{n}^{(s)}=\lambda_{n}^{(j)}\right\}\right\} .
$$

Proof. To prove the theorem, consider $\rho_{n}(z)=n+\frac{z}{n \pi},|z| \leqslant R$. Substituting $\rho=\rho_{n}(z)$ into $(9)-(12)$, we obtain

$$
\begin{aligned}
S_{j}\left(\pi, \rho_{n}^{2}(z)\right)= & \frac{(-1)^{n}}{n \rho_{n}(z)}\left(z-\tilde{\omega}_{j n}+\frac{\kappa_{n}(z)}{n}\right), \quad \tilde{\omega}_{j n}=\omega_{j}-\widehat{q}_{j}(2 n), \\
& S_{j}^{\prime}\left(\pi, \rho_{n}^{2}(z)\right)=(-1)^{n}\left(1+\frac{\kappa_{n}(z)}{n}\right), \\
& C_{j}\left(\pi, \rho_{n}^{2}(z)\right)=(-1)^{n}\left(1+\frac{\kappa_{n}(z)}{n}\right), \\
C_{j}^{\prime}\left(\pi, \rho_{n}^{2}(z)\right)= & \frac{(-1)^{n} \rho_{n}(z)}{n}\left(z-\breve{\omega}_{j n}+\frac{\kappa_{n}(z)}{n}\right), \breve{\omega}_{j n}=\omega_{j}+\widehat{q}_{j}(2 n),
\end{aligned}
$$

where $\widehat{q}_{j}(l)=\frac{1}{2} \int_{0}^{\pi} q_{j}(t) \cos l t d t$. We substitute (21)-(24) into (14), (18) and get

$$
\Delta\left(\rho_{n}^{2}(z)\right)=\frac{(-1)^{n m}}{n^{m-1} \rho_{n}^{m-1}(z)}\left(\sum_{\substack{s=1 \\ j}}^{m} \prod_{\substack{j=1 \\ j \neq s}}^{m}\left(z-\tilde{\omega}_{j n}\right)+\frac{\kappa_{n}(z)}{n}\right),
$$




$$
M_{k k}\left(\rho_{n}^{2}(z)\right) \Delta\left(\rho_{n}^{2}(z)\right)=\frac{(-1)^{n m}}{n^{m-2} \rho_{n}^{m-2}(z)}\left(\sum_{\substack{s=1, s \neq k \\ s \neq k=1, j \neq k}}^{m} \prod_{\substack{j=1, j \neq s, j}}^{m}\left(z-\tilde{\omega}_{j n}\right)+\frac{\kappa_{n}(z)}{n}\right) .
$$

Let us denote $f_{n}(z)=\prod_{j=1}^{m}\left(z-\tilde{\omega}_{j n}\right), \delta(r)$ is the circle of center 0 and radius $r>0$. It can be proved that $\tilde{z}_{n}^{(j)}=z^{(j)}+o(1), n \rightarrow \infty$, where $\left\{\tilde{z}_{n}^{(j)}\right\}_{j=1}^{m-1}$ are the zeros of $f_{n}^{\prime}(z)$. If $z \in \delta(R)$, then for sufficiently large $n, \rho_{n}^{2}(z)$ runs across the simple closed contour, which surrounds $\left\{\lambda_{n}^{(j)}\right\}_{j=1}^{m-1}$. Integrating $M_{k k}(\lambda)$, after the substitution $\lambda=\rho_{n}^{2}(z)$ we have

$$
\sum_{l \in I(n)} \alpha_{l n}^{k}=\frac{1}{2 \pi i} \int_{z \in \delta(R)} \frac{2 \rho_{n}(z)}{n \pi} M_{k k}\left(\rho_{n}^{2}(z)\right) d z .
$$

The following formula is obtained from the previous one and (25), (26):

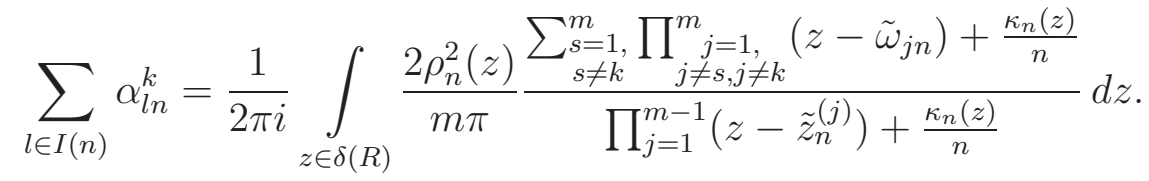

The remainder $\frac{\kappa_{n}(z)}{n}$ can be excluded from the denominator of (27) with Taylor expansion as $\min _{|z|=R} \prod_{j=1}^{m-1}\left|z-\tilde{z}_{n}^{(j)}\right|>1$ if $n$ is large enough. Besides, $\rho_{n}^{2}(z)=n^{2}\left(1+\frac{\kappa_{n}(z)}{n}\right)$, $|z| \leqslant R$. After the designation

$$
g_{k n}(z)=\frac{\sum_{\substack{s=1 \\ s \neq k}}^{m} \prod_{\substack{j=1, j \neq s, j \neq k}}^{m}\left(z-\tilde{\omega}_{j n}\right)}{\prod_{j=1}^{m-1}\left(z-\tilde{z}_{n}^{(j)}\right)}
$$

we get

$$
\sum_{l \in I(n)} \alpha_{l n}^{k}=\frac{2 n^{2}}{2 m \pi^{2} i}\left(\int_{z \in \delta(R)} g_{k n}(z) d z+\frac{\kappa_{n}}{n}\right)
$$

We note that $\delta(r)$ contains all $\left\{\tilde{z}_{n}^{(j)}\right\}_{j=1}^{m-1}$ for $r \geqslant R$ and large $n$. Thus,

$$
\int_{z \in \delta(R)} g_{k n}(z) d z=\int_{z \in \delta(r)} g_{k n}(z) d z .
$$

The numerator of the fraction $g_{k n}(z)$ is a polynomial of degree $m-2$ with leading coefficient $m-1$, and its denominator is a polynomial of degree $m-1$ with leading coefficient 1 . For $z \in \delta(r)$ there is the equality $g_{k n}(z)=\frac{m-1}{z}+O\left(r^{-2}\right)$, and

$$
\frac{1}{2 \pi i} \int_{z \in \delta(r)} g_{k n}(z) d z=m-1+O\left(r^{-1}\right) .
$$

As $r \rightarrow \infty$ we obtain (19).

Formula (20) is proved analogously. 
Theorem 3. Let $z^{(s)}$ be a zero of $f^{\prime}(z)$ of multiplicity $b(s)>0,1 \leqslant p \leqslant m$. Denote $N(s)=\left\{1 \leqslant j<m: z^{(s)} \neq z^{(j)}\right\}, N^{\prime}(s)=\left\{1 \leqslant j<m: z^{(s)}=z^{(j)}\right\}$, and $W(s)=\left\{1 \leqslant j \leqslant m: z^{(s)} \neq \omega_{j}\right\}$. If $p \in W(s)$, then

$$
\sum_{l \in N^{\prime}(s)} \alpha_{l n}^{p}=\frac{2 n^{2}}{m \pi}\left(\Omega_{p s}+\kappa_{n}\right)
$$

else

$$
\sum_{l \in N^{\prime}(s)} \alpha_{l n}^{p}=\frac{2 n^{2}}{m \pi}\left(\Theta_{s}+\kappa_{n}\right)
$$

where

$$
\Omega_{p s}=-\frac{\prod_{j=1}^{m}\left(z^{(s)}-\omega_{j}\right)}{\left(z^{(s)}-\omega_{p}\right)^{2} \prod_{j \in N(s)}\left(z^{(s)}-z^{(j)}\right)}, \quad \Theta_{s}=b(s) \frac{\prod_{j \in W(s)}\left(z^{(s)}-\omega_{j}\right)}{\prod_{j \in N(s)}\left(z^{(s)}-z^{(j)}\right)},
$$

and the product over empty set is understood as 1 .

Proof. Denote by $r$ such positive number that the circle $\left|z-z^{(s)}\right| \leqslant r$ does not contain $z^{(j)}, j \in N(s)$ and $\left|z^{(s)}\right|+r<R, r \geqslant C>0$. We call the circumference of that circle $\gamma(s)$. The following analogue of the formulae (28) can be proved:

$$
\sum_{l \in N^{\prime}(s)} \alpha_{l n}^{p}=\frac{2 n^{2}}{2 m \pi^{2} i}\left(\int_{\gamma(s)} \frac{\sum_{\substack{k=1 \\ k \neq p}}^{m} \prod_{\substack{j \neq k=1, j \\ j \neq p}}^{m}\left(z-\tilde{\omega}_{j n}\right)}{\prod_{j=1}^{m-1}\left(z-\tilde{z}_{n}^{(j)}\right)} d z+\frac{\kappa_{n}}{n}\right) .
$$

We designate

$$
F_{p}(z)=\frac{\sum_{\substack{k=1, k \neq p}}^{m} \prod_{\substack{j \neq k, j \neq p \\ j \neq 1,}}^{m}\left(z-\omega_{n}\right)}{\prod_{j=1}^{m-1}\left(z-z^{(j)}\right)} .
$$

As $\omega_{j}-\tilde{\omega}_{j n}=\kappa_{n}$ and the coefficients of $f^{\prime}(z), f_{n}^{\prime}(z)$ depend on $\left\{\omega_{j}\right\}_{j=1}^{m},\left\{\tilde{\omega}_{j n}\right\}_{j=1}^{m}$ polynomially, we have

$$
\frac{\sum_{\substack{k=1, k \neq p}}^{m} \prod_{\substack{j=1, j \neq k, j \neq p}}^{m}\left(z-\tilde{\omega}_{j n}\right)}{\prod_{j=1}^{m-1}\left(z-\tilde{z}_{n}^{(j)}\right)}-F_{p}(z)=\kappa_{n}(z),
$$

where $z \in \gamma(s)$. We integrate the fraction $F_{p}(z)$.

First we consider $b(s)>1$. Then $z^{(s)}$ is a zero of $f(z)$ of multiplicity $b(s)+1$ (see [10, section 4.3]), and cardinality of $W(s)$ is $m-b(s)-1$. In the case when $p \in W(s)$ the function $F_{p}(z)$ has no pole inside $\gamma(s)$, and $\alpha_{s n}^{p}=\frac{2 n^{2}}{m \pi} \kappa_{n}$, what is the same as (29). If $p \notin W(s)$, then

$$
F_{p}(z)=\frac{b(s)\left(z-z^{(s)}\right)^{b(s)-1} \prod_{j \in W(s)}\left(z-\omega_{j}\right)+\left(z-z^{(s)}\right)^{b(s)} \sum_{k \in W(s)} \prod_{\substack{j \in W(s) \\ j \neq k}}\left(z-\omega_{j}\right)}{\left(z-z^{(s)}\right)^{b(s)} \prod_{j \in N(s)}\left(z-z^{(j)}\right)},
$$

and

$$
\int_{\gamma(s)} F_{p}(z) d z=b(s) \frac{\prod_{j \in W(s)}\left(z^{(s)}-\omega_{j}\right)}{\prod_{j \in N(s)}\left(z^{(s)}-z^{(j)}\right)} .
$$

Formula (30) follows from (31)-(33). 
Further, let $b(s)=1$. When $z^{(s)}$ is a zero of $f(z)$, computations are the same as in the case $b(s)>1$. So we assume $f\left(z^{(s)}\right) \neq 0$, and consequently $p \in W(s)$. Rewriting $F_{p}(z)$ as

$$
F_{p}(z)=\left(\frac{f(z)}{z-\omega_{p}}\right)^{\prime}\left(f^{\prime}(z)\right)^{-1}=\frac{1}{z-\omega_{p}}-\frac{f(z)}{\left(z-\omega_{p}\right)^{2} f^{\prime}(z)}
$$

and integrating over $\gamma(s)$, we obtain (30).

Acknowledgements: This work was supported in part by the Russian Foundation for Basic Research (projects nos. 15-01-04864, 17-51-53180) and by the Ministry of Education and Science of the Russian Federation (project no. 1.1660.2017/PCh).

\section{References}

1. Yang C.-F., Huang Z.-Y., Yang X.-P. Trace formulas for Schrödinger systems on graphs. Turkish J. Math., 2010, vol. 34, no. 2, pp. 181-196. DOI: 10.3906/mat-0811-7.

2. Berkolaiko G., Kuchment P. Introduction to Quantum Graphs. AMS, Providence, RI, 2013. 270 p.

3. Pokorny Yu. V., Penkin O. M., Borovskikh A. V., Pryadiev V. L., Lazarev K. P., Shabrov S. A. Differentsial'nye uravneniia na geometricheskikh grafakh [Differential Equations on Geometrical Graphs]. Moscow, Fizmatlit, 2004. 272 p. (in Russian).

4. Freiling G., Yurko V. A. Inverse Sturm-Liouville problems and their applications. New York, Nova Science, 2001. 305 p.

5. Yurko V. A. On recovering Sturm-Liouville operators on graphs. Math. Notes, 2006, vol. 79 , iss. $3-4$, pp. 572-582. DOI: $10.4213 / \mathrm{mzm} 2732$.

6. Yurko V. A. Inverse spectral problems for differential operators on spatial networks. Russian Math. Surveys, 2016, vol. 71, no. 3, pp. 539-584. DOI: 10.4213/rm9709.

7. Bondarenko N. Spectral analysis for the matrix Sturm - Liouville operator on a finite interval. Tamkang J. Math., 2011, vol. 42, no. 3, pp. 305-327. DOI: 10.5556/j.tkjm.42.2011.305327.

8. Pivovarchik V. Inverse problem for the Sturm - Liouville equation on a star-shaped graph. Math. Nachr., 2007, vol. 280, no. 1314, pp. 1595-1619. DOI: 10.1002/mana.200410567.

9. Möller M., Pivovarchik V. Spectral theory of operator pencils, Hermite-Biehler functions, and their applications. Cham, Birkhäuser, 2015. 412 p. DOI: 10.1007/978-3-31917070-1.

10. Hardy G. H., Littlewood J. E., Polya G. Inequalities. London, Cambridge University Press, 1934. $456 \mathrm{p}$.

\section{Cite this article as:}

Kuznetsova M. A. Asymptotic Formulae for Weight Numbers of the Sturm - Liouville Boundary Problem on a Star-shaped Graph. Izv. Saratov Univ. (N. S.), Ser. Math. Mech. Inform., 2018, vol. 18, iss. 1, pp. 40-48. DOI: 10.18500/1816-9791-2018-18-1-40-48. 


\title{
УДК 517.984
}

\section{АСИМПТОТИЧЕСКИЕ ФОРМУЛЫ ДЛЯ ВЕСОВЫХ ЧИСЕЛ КРАЕВОЙ ЗАДАЧИ ШТУРМА - ЛИУВИЛЛЯ НА ГРАФЕ-ЗВЕЗДЕ}

\begin{abstract}
М. А. Кузнецова
Кузнецова Мария Андреевна, студент, Саратовский национальный исследовательский государственный университет имени Н. Г. Чернышевского, 410012, Россия, Саратов, Астраханская, 83, mk680970@gmail.com

В статье исследована краевая задача Штурма - Лиувилля на грасре Г определенного вида. Граср Г имеет $m$ ребер, смежных с одной внутренней вершиной, а остальные $m$ вершин являются вершинами степени 1. Краевая задача на данном грасре задается дифрференциальными выражениями Штурма - Лиувилля с вещественными потенциалами, краевыми условиями Дирихле и стандартными условиями склейки. Определенная таким образом краевая задача имеет счетное множество собственных значений. Мы рассмотрим вычеты диагональных элементов матрицы Вейля в собственных значениях, которые назовем весовыми числами. Элементы матрицы Вейля являются мероморсрными фрункциями с простыми полюсами в собственных значениях. Отметим, что весовые числа в данном случае являются обобщением весовых чисел оператора Штурма - Лиувилля на конечном интервале, которые определяются как обратные величины квадратов норм собственных фрункций. Эти числа вместе с собственными значениями играют роль спектральных данных для однозначного восстановления оператора. С помощью интегрирования по контурам будут получены асимптотические фрормулы для весовых чисел, в случае асимптотически близких собственных значений будем иметь фрормулы для сумм. Результаты могут быть использованы для анализа обратных спектральных задач на грасрах.

Ключевые слова: краевая задача Штурма-Лиувилля, асимптотические срормулы, весовые числа, графр-звезда.
\end{abstract}

Благодарности. Работа выполнена при частичной финансовой поддержке РФФИ (проекты № 15-01-04864, 17-51-53180) и Минобрнауки РФ (проект № 1.1660.2017/ПЧ).

\section{Образец для цитирования:}

Кузнецова M. A. Asymptotic Formulae for Weight Numbers of the Sturm-Liouville Boundary Problem on a Star-shaped Graph [Асимптотические формулы для весовых чисел краевой задачи Штурма - Лиувилля на графе-звезде] // Изв. Сарат. ун-та. Нов. сер. Сер. Математика. Механика. Информатика. 2018. Т. 18, вып. 1. С. 40-48. DOI: 10.18500/1816-9791-2018-18-140-48. 